\title{
Opportunistic Contention-Based Feedback Protocol for Downlink OFDMA Systems with Mixed Traffic
}

\author{
Rachod Patachaianand, Kumbesan Sandrasegaran, \\ Huda Adibah Mohd Ramli, and Riyaj Basukala \\ Faculty of Engineering and IT, University of Technology Sydney, \\ NSW 2007, Australia \\ \{rapatcha, kumbes, HudaAdibah. MohdRamli\}@eng.uts.edu.au, \\ Riyaj.Basukala@uts.edu.au
}

\begin{abstract}
An opportunistic feedback protocol is proposed for orthogonal frequency division multiple access (OFDMA) systems with mixed realtime (RT) and non-realtime (NRT) traffic. This protocol allocates a dedicated feedback channel for each RT user, while it allows all NRT users to compete to send feedback via a contention-based random access feedback channel. The proposed feedback protocol requires a fixed amount of resource to carry feedback from all RT users and some selected NRT users. The probability that NRT users attempt to send feedback is based on two parameters: a channel quality threshold, and the number of subcarriers (or clusters) whose channel quality is above the threshold. Simulation results demonstrate that allocating a dedicated feedback channel for RT user improves the achievable throughput significantly compared with allocating a dedicated traffic channel. For NRT users, the achievable sum rate of the proposed protocol when the number of users is moderate (20-50 users) approaches the ideal case when the channel quality of all users is known at the base station. The sum rate also is close to the ideal case when the number contention minislots per subcarrier is as low as 2 minislot per subcarrier.
\end{abstract}

Keywords: Opportunistic scheduling, Limited feedback, Contention, OFDMA.

\section{Introduction}

Next generation wireless networks will be designed to support a variety of multimedia applications which require different Quality-of-Service (QoS). Orthogonal frequency division multiple access (OFDMA) has been chosen as the radio access technology to support such high requirement applications in WiMAX and Long Term Evolution (LTE) systems. In OFDMA systems, all users share the radio resource which comprises a number of clusters of subcarriers. It has been shown that optimally allocating the subcarriers (or clusters) to users based on their instantaneous channel state information (CSI) can improve the performance of the system significantly.

Various approaches have been proposed to minimise the amount of CSI feedback. In [1], a feedback reduction protocol where all users compete to send feedback via a 

share contention-based feedback channel is proposed. The idea of the contentionbased feedback in [1] is to have a number of minislots in the uplink feedback channel for users to compete to send feedback messages. The users whose channel quality exceeds a threshold compete for one of the minislots with some predetermined probability. The same idea was extended for OFDMA systems in [2]. The use of contention-based feedback was extended in [3] by dividing users into multiple groups each of which has a separate set of feedback minislots. In [4], an OFDMA resource allocation with multiple-threshold contention-based feedback protocol was proposed. In [5], another contention-based feedback protocol with multiple threshold in which users compete for feedback minislots based on their channel quality was introduced.

In this paper, we propose a new protocol to reduce the amount of the feedback resources needed for OFDMA systems with mixed realtime (RT) and non-realtime (NRT) traffic. To better explain the contribution of our work, let us consider an OFDMA system supporting $K$ users with $N$ subcarriers, and assume that the total number of feedback minislots is $X$. With other contention-based feedback protocols such as in [2][5], $X / N$ feedback minislots will be assigned for each subcarrier, and thus the probability of successful contention decreases and contention is likely to occur since the number of minislots is small. With our proposed protocol, in contrast, all $X$ minislots are available for NRT users to compete to send feedback. We also introduce a new selection criterion to optimally minimise the number of users sending feedback by allowing only NRT users whose channel quality is above the threshold in a number of subcarriers to attempt to send feedback. We prove through simulations that allowing NRT users to compete on $X$ minislots provides a significant performance gain over allowing them to access $X / N$ minislots on each subcarrier.

The remainder of this paper is organised as follows: The system model is given in Section 2. Section 3 describes the proposed feedback protocol. Simulation results are discussed in Section 4. Section 5 concludes this work.

\section{System Model}

We consider the downlink data transmission of an OFDMA system having $N$ subcarriers. The total transmit power is constant at $P_{t}$. A frequency-selective Rayleigh fading channel is considered. It is assumed that the channel gains for all users are independent and identically distributed (i.i.d.) for all subcarriers. For user $k$ on subcarrier $n$, the channel gain $h_{k n}$ is assumed to be perfectly known at the respective receiver, and the transmitter knows the channel gains by CSI sent from the receivers only. The received signal-to-noise ratio (SNR) as $\gamma_{k n}$. All users are assumed to have homogeneous SNR. The feedback links are assumed to be delay-free and error-free.

\section{Proposed Feedback Protocol}

Each subcarrier contains $M$ contention-based minislots, which implies $M \times N$ minislots in total. The NRT users need to receive a CSI transmission grant message sent from BS to allow them to send CSI. To obtain the grant message, the users need to compete 
for a contention-based minislot. We assume that all users can compete for the entire $M \times N$ minislots. The contention may occur on these $M \times N$ minislots only.

After the $M$ contention-based minislot, there is a non-contention-based CSI feedback minislot which is provided for a user who successfully competes for a contention minislot to send CSI feedback (see Fig 1).

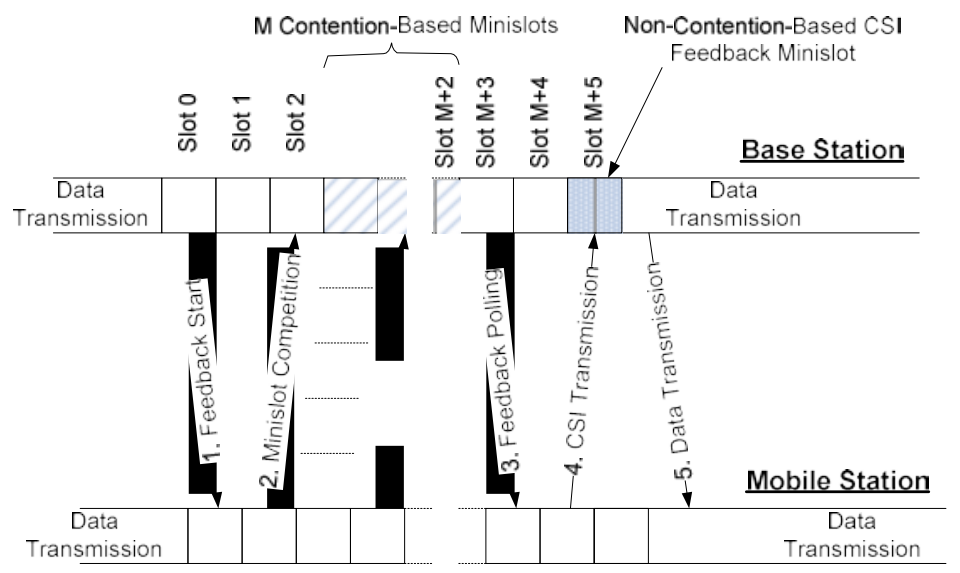

Fig. 1. The proposed feedback procedure. BS and MSs are perfectly synchronised

We assume that the message to be sent on the contention minislot is user ID while the information to be sent on the non-contention minislot is the CSI. Each CSI feedback minislot can carry only one CSI message from one user. Therefore, if the number of the users who successfully obtain a contention minislot exceeds the number of CSI feedback channels, the BS randomly selects $N$ users out of the users who successfully compete for a minislot to send their user ID to allow sending CSI feedback.

The steps involved in the proposed protocol as shown in Fig 1 are explained below:

\section{Step 1: Feedback Start}

The BS broadcasts a feedback message containing information regarding the opportunistic feedback to all mobile stations (MSs). This message includes $N_{t h}$ and $\gamma_{t h}$ parameters that will be discussed in details in Step 2.

\section{Step 2: Minislot Competition}

Once MSs received the broadcast massage, they start competing for feedback minislots. To optimally allow users to attempt to compete for a feedback minislot, a new feedback selection criterion is introduced. We define $\beta_{k}$ as the number of subcarriers having channel quality above the threshold for user $k$ :

$$
\beta_{k}=\sum_{n=1}^{N} U\left(\gamma_{k n}-\gamma_{t h}\right)
$$

where $\gamma_{\text {th }}$ is the channel quality threshold, $U(x)=1$ when $x>0$, and $U(x)=0$ when $x$ $\leq 0$. User $k$ is allowed to attempt to compete for a minislot if and only if $\beta_{k}>N_{t h}$ 
where $N_{\text {th }}$ is the minimum number of subcarriers on which signal quality is above the threshold $\gamma_{t h}$. Only those users having channel quality above a predefined threshold on a number of subcarriers are permitted to attempt competing for a minislot to send feedback. If two or more users attempt to send feedback on the same feedback minislot, there is a collision and the feedback is assumed to be irrecoverable.

\section{Step 3: Feedback Polling}

After the contention phase is finished, the BS knows the number of users who successfully compete for the minislots and their user IDs. The BS then selects $N$ (= $N_{R T}+N_{N R T}$ ) users out of the successful users in random and broadcasts a polling message to all users to identify which users are allowed to send CSI. Note that all RT users are always included in the feedback polling message.

\section{Step 4: CSI Feedback Transmission}

Those users whose user ID is in the polling message send CSI to the BS. In this work, the Max-Q feedback reduction scheme [6] is used for both RT and NRT users. This algorithm allows each user to send a CSI message containing the SNR levels of $Q$ highest subcarriers. Since CSI information of $Q$ subcarriers is sent, the system can guarantee the minimum resource for $N_{R T}<Q$ RT users. This is because, in a rare circumstance, all $N_{R T}$ users may have the same set of $Q$ best subcarriers.

\section{Step 5: Data Transmission}

Once the BS received CSI from all N users, it performs subcarrier allocation based on the received CSI. A simple subcarrier allocation where each subcarrier is allocated to a user having the highest gain among all users on this subcarrier is used in this paper. The achievable average capacity for subcarrier $n$ thus can be given by:

$$
C_{n}=E\left\{\log _{2}\left(1+\max _{k^{\prime}} \gamma_{k^{\prime} n_{k^{\prime}}}\right)\right\}
$$

where $k$ ' represents the set of users who successfully send CSI feedback and $n_{k}$ ' represents the set of index of $Q$ subcarriers having highest SNR of the $k^{\text {th }}$ user. $E\{x\}$ denotes the expectation value of random variable $x$.

\section{Simulation Results}

In this section, we focus on the performance of the proposed scheme using the achievable average sum rate, and then compare with the feedback protocol proposed in [2]. Simulations were performed using MATLAB-based Monte Carlo simulations using an average SNR for all users fixed at $6 \mathrm{~dB}, Q=5, N=32$, and $N_{t h}=10$ unless mentioned otherwise. Each RT user requires a minimum of one subcarrier to maintain the session, while NRT users are served on a best-effort basis. We assume that both RT and NRT always have data to be sent.

\subsection{Performance of RT Users}

With the proposed protocol, BS always allows RT users to send CSI with no collision. Once BS received the CSI from RT users, it provides higher priority for RT in the 
subcarrier allocation. This means each RT user will be allocated its best subcarrier with high probability. The only chance that capacity loss may occur is when different RT users have the same subcarriers having highest SNR. However, the probability of this event is very low and thus the loss can be neglected.

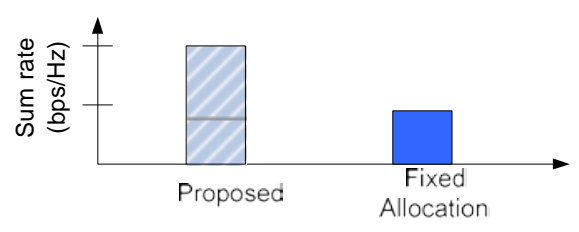

Fig. 2. Achievable sum-rate for the dedicated feedback and dedicated traffic channels

Fig 2 shows that the proposed scheme can achieve much higher performance in terms of achievable sum rate than the fixed allocation because the proposed scheme transmits the signal on the subcarrier that has the highest SNR, thus having benefit from frequency diversity. However, this performance gain increases the feedback load. Assuming a CSI report consists of $B$ bits, the feedback load of the fixed scheme per each RT user can be expressed as: $F B_{\text {Fixed }}=B+D$ when $D$ is the total number of bits for user ID. The CQI bits required for our proposed scheme is given by [6]:

$$
F B_{\mathrm{Pr}_{\text {oposed_RT }} R}=B \cdot Q+\log _{2}\left(\begin{array}{l}
N \\
Q
\end{array}\right)+D
$$

The different between $F B_{\text {Fixed }}$ and $F B_{\text {Proposed_RT }}$ is given in Table 1.

Table 1. Difference in Feedback Load $(B=5)$

\begin{tabular}{|l|l|l|l|l|l|l|l|l|l|l|}
\hline $\mathrm{Q}$ & $\mathbf{1}$ & $\mathbf{2}$ & $\mathbf{3}$ & $\mathbf{4}$ & $\mathbf{5}$ & $\mathbf{6}$ & $\mathbf{7}$ & $\mathbf{8}$ & $\mathbf{9}$ & $\mathbf{1 0}$ \\
\hline$\triangle \mathrm{FB}$ & 5 & 14 & 23 & 31 & 38 & 45 & 52 & 59 & 65 & 71 \\
\hline
\end{tabular}

From Tabel 1 , for $Q=1$, the proposed scheme requires 5 extra bits which are needed for identifying the subcarrier index of the highest-SNR subcarrier. The choice of $Q$ has a significant impact on the achievable sum rate of NRT services especially when the number of users is small (see [6] for detail).

\subsection{Performance of NRT Users}

Fig. 3 demonstrates that the performance of the proposed protocol is close to the ideal case for a moderate number of active users (20-50 NRT users). Overall, the proposed scheme significantly outperforms the scheme in [2] over a wide range of users because allowing NRT users to compete for a larger number of minislots increase the probability of successful feedback. 


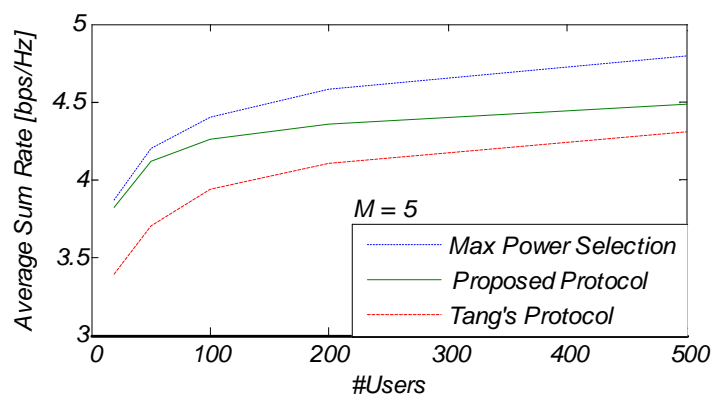

Fig. 3. The average sum-rate versus the number of users $K$ (NRT)

\section{Conclusion}

In this paper, we propose a new feedback protocol for OFDMA systems with mixed RT and NRT traffic. For RT users, a dedicated CSI feedback channel is allocated. For NRT, the protocol is based on the contention-based feedback resource where all NRT users can attempt to send feedback via feedback minislots on any subcarriers. The NRT users are allowed to attempt for a feedback minislot only if their number of subcarriers whose channel quality above the threshold is greater than a predefined threshold value. Simulation results demonstrate that, for RT case, assigning a dedicated CSI feedback channel for each RT user provides a significant performance gain over allocating a fixed subcarrier. For NRT users, the proposed scheme can achieve a large proportion of the achievable average sum-rate even if only two feedback minislots per subcarrier are allowed. The performance of the proposed scheme is close to the maximum power allocation scheme especially when the number of users is small. The simulation results also illustrate that allowing users to compete on all feedback minislots over entire subcarriers increase the probability of successful feedback and thus improves the performance of the system.

\section{References}

1. Tang, T., Heath, R.: Opportunistic Feedback for Downlink Multiuser Diversity. IEEE Comm. Letters 9, 948-950 (2005)

2. Tang, T., Heath, R., Cho, S., Yun, S.: Opportunistic Feedback in Clustered OFDM Systems. In: International Symposium on Wireless personal Multimedia Communications, San Diego, CA (September 2006)

3. Patil, S., Veciana, G.: Feedback and Opportunistic Scheduling in Wireless Networks. IEEE Trans. Wireless Commun. 6(12), 4227-4238 (2007)

4. Agarwal, R., Majjigi, V., Han, Z., Vannithamby, R., Cioffi, J.: Low Complexity Resource Allocation with Opportunistic Feedback over Downlink OFDMA Networks. IEEE JSAC Special Issue on Limited Feedback 26(8), 1462-1472 (2008)

5. Jaewoo, S., Cioffi, J.: Feedback Reduction Scheme for Downlink Multiuser Diversity. IEEE Trans. Wireless Commun. 8(2), 668-672 (2009)

6. Kolehmainen, N., Puttonen, J., Kela, P., Ristaniemi, T.: Channel Quality Indication Reporting Schemes for UTRAN Long Term Evolution Downlink. In: Vehicular Technology Conference, Marina Bay, Singapore, May 11-14, pp. 2522-2526 (2008) 